\title{
ENSAIOS DE COMBATE AO CUPIM DE MONTE Cornitermes cumulans (KOLLAR, 1832) (ISOPTERA, TERMITIDAE)
}

\author{
F.A.M. MARICONI; V.B. GALAN; M.T. ROCHA; R.F. MAULE; H.R. PASSOS; R.A.A. SILVA \\ Departamento de Zoologia-ESALQ/USP, C.P. 9 - CEP: 13418-900 - Piracicaba,SP
}

\begin{abstract}
RESUMO: Com a finalidade de combater o cupim de monte Cornitermes cumulans, importante espécie invasora, foram instalados dois campos experimentais. Experimento I: 60 ninhos foram escolhidos e medidos externamente. $O$ ensaio constou de 6 tratamentos com 10 repetiçzes: A) abamectina (50 $\mathrm{cm}^{3} \mathrm{CE} \mathrm{1,8 \% );} \mathrm{B)} \mathrm{silafluofem}\left(200 \mathrm{~cm}^{3} \mathrm{CE}\right.$ $80 \%$ ); C) silafluofem (400 $\mathrm{cm}^{3} \mathrm{CE} \mathrm{80 \% );} \mathrm{D)} \mathrm{fipronil} \mathrm{(15g} \mathrm{G} \mathrm{2 \% );} \mathrm{E)} \mathrm{fipronil} \mathrm{(20g} \mathrm{G} \mathrm{2 \% );} \mathrm{F)} \mathrm{clorpirifós} \mathrm{(30g} \mathrm{G}$ $0,125 \%)$. Nos tratamentos $A, B, C$, as quantidades entre parênteses são da formulação comercial em 100 litros de água. Em D,E,F, são do granulado para cada nimho. Dos líquidos, usou-se um litro de calda por monte. A demolição dos montes deu-se ap6s 103 dias da aplicação. Os melhores resultados foram obtidos com a abamectina e fipronil. Experimento II: Também neste ensaio os cupinzeiros foram 60, abrangendo 6 tratamentos com 10 repetições: A) fipronil (10g G 2\%); B) fipronil (15g G 2\%); C) bendiocarbe $(20 \mathrm{~g} \mathrm{G} \mathrm{0,1 \% );} \mathrm{D)} \mathrm{bendiocarbe}(20 \mathrm{~g} \mathrm{G} \mathrm{0,5 \% );} \mathrm{E)}$ imidaclopride $(0,15 \mathrm{~g}$ G 70\%); F) imidaclopride $(0,30 \mathrm{~g} \mathrm{G} \mathrm{70 \% ).} \mathrm{Em} A, B, C, D$, as quantidades de granulados são por ninho. Em E,F são de granulado dispersível em um litro de água, por ninho. A destruição dos cupinzeiros foi feita 148 e 149 dias ap6s a aplicação. Os melhores resultados foram o fipronil e imidaclopride.
\end{abstract}

Descritores: cupim de monte, Cornitermes cumulans, inseticidas granulados

\section{FIELD TESTS FOR CONTROL OF THE MOUND-BUILDING TERMITE Cormitermes cumulans (KOLLAR, 1832) (ISOPTKRA, TERMITIDAE)}

\begin{abstract}
ARSTRACT: Two field tests were carried out to evaluate the performance of several pesticides for the control of the mound termite pest in pastures. Experiment I: 60 mounds were selected and measured outside. There were 6 treatments with 10 replications: A) abamectin $\left(50 \mathrm{~cm}^{3} 1.8 \% \mathrm{EC}\right)$; B) silafluofen $\left(200 \mathrm{~cm}^{3} 80 \% \mathrm{EC}\right)$; C) silafluofen $\left(400 \mathrm{~cm}^{3} 80 \% \mathrm{EC}\right)$; D) fipronil (15g $\left.2 \% \mathrm{G}\right)$; E) fipronil (20g $\left.2 \% \mathrm{G}\right)$; F) chlorpirifos $(30 \mathrm{~g} 0.125 \% \mathrm{G})$. In A,B,C, the quantities between parenthesis are of the commercial formulation in 100 liters of water. In D,E,F, are of granular insecticides per mound. One liter of the liquids was used per nest. Demolition of the mounds were made 103 days after the application. The most efficient were abamectin and fipronil. Experiment II: Also 60 nests, with 6 treatments and 10 replications: A) fipronil $(10 \mathrm{~g} 2 \% \mathrm{G})$; B) fipronil $(15 \mathrm{~g} 2 \% \mathrm{G})$; C) bendiocarb $(20 \mathrm{~g} 0.1 \% \mathrm{G})$; D) bendiocarb $(20 \mathrm{~g} 0.5 \% \mathrm{G}) ; \mathrm{E})$ imidacloprid $(0.15 \mathrm{~g} 70 \% \mathrm{G})$; F) imidacloprid $(0.30 \mathrm{~g} 70 \% \mathrm{G})$. In $\mathrm{A}, \mathrm{B}, \mathrm{C}, \mathrm{D}$, the quantities of gramular insecticides are by nest. In E,F, of dispersible granule in 1 liter of water, by nest. The demolition of the nests was made 148 and 149 days after the application. The most efficient were fipronil and imidacloprid.
\end{abstract}

Key Words: mound termite, Cornitermes cumulans, granular insecticides

\section{INTRODUÇÃO}

O cupim de monte Cornitermes cumulans é, dentre as espécies de cupins de monte, a mais comum no Estado de São Paulo e estados vizinhos. Seu ninho, em forma de monte, aflora à superfície do solo dificultando ou impossibilitando o manejo de máquinas e qualquer tipo de preparo de solo. $O$ estudo do combate a esta praga tem sido feito por vários autores. Os produtos utilizados em trabalhos mais antigos foram, em grande parte, retirados do mercado. VANETTI (1959) realizou ampla experi- mentação tendo apenas encontrado três tratamentos com resultados satisfatórios. AMANTE (1963) obteve bons resultados com apenas um produto, embora tenha testado vários e em 3 diferentes dosagens. Bons resultados, com alguns produtos, foram conseguidos por NAKANO \& PROSPERO (1969). Trabalhando com 210 ninhos, MARICONI et al. (1971) conseguiram ótimos resultados, mas a maioria dos produtos utilizados está fora do mercado; foi demonstrado que, após a aplicação do inseticida, não há necessidade de fechar-se o canal de aplicação. SUGAHARA et al. (1987) 
verificaram a eficiência do fentiom: com $1,25 \mathrm{~g}$ de ingrediente ativo (IA) por ninho a mortalidade foi de $80 \%$ e com 2,5g foi de $100 \%$. MOTTA et al. (1987) obtiveram $100 \%$ de mortalidade com somente $1,0 \mathrm{~g}$ de IA por ninho. BIONDO et al. (1988) conseguiram também $100 \%$ de mortalidade com fentiom e $90 \%$ com a abamectina. MARICONI et al. (1989) usaram quatro dosagens de clorpirifós e duas de fentiom, obtendo $100 \%$ de mortalidade com todas elas. HAMAMURA et al. (1990), dentre os tratamentos, havia um que era somente água (testemunha). Dos 10 ninhos submetidos à água, apenas 1 morreu. MARICONI et al. (1990) verificaram que a água não interferiu; todos os ninhos estavam bem vivos na ocasião da demolição.

\section{MATERIAIS E MÉTODOS}

\section{Experimento I}

Local: Propriedade rural localizada no bairro Tuncum, município de São Pedro, SP, de propriedade do Sr. Luiz Bucher. Cupinzeiros localizados em pastagem.

Tratamentos: Seis, com dez repetições, cada uma destas constituídas por um cupinzeiro. Ao total foram utilizados 60 ninhos, todos em pastagem. A altura média por tratamento foi: A) $0,69 \mathrm{~m}$; B)
$0,62 \mathrm{~m}$; C) $0,67 \mathrm{~m}$; D) $0,60 \mathrm{~m}$; E) $0,76 \mathrm{~m}$; F) $0,65 \mathrm{~m}$. A média das alturas dos tratamentos foi $0,67 \mathrm{~m}$. Ver produtos, formulações, concentrações e consumo de material na TABELA 1.

\begin{abstract}
Abertura de canal: Utilizou-se uma barra de aço de $25 \mathrm{~mm}$ de diâmetro e cerca de 60 a $70 \mathrm{~cm}$ de comprimento, que, batida com pesada marreta, atingisse $o$ núcleo.
\end{abstract}

Aplicação: Realizada em 26 de fevereiro de 1993. Em A,B,C, cada ninho recebeu 1 litro de calda, de maneira que atingisse diretamente $O$ núcleo. Os tratamentos D, E e F foram granulados, portanto, aplicados a seco.

Destruição dos ninhos: Realizada em 10 de junho de 1993 (após 103 dias da aplicação). Não havendo presença de $C$. cumulans o ninho era considerado morto e, caso contrário, vivo.

\section{Experimento II}

Local: pastagem localizada em sítio do sr. José Mandro, bairro do Serrote, município de Piracicaba, Estado de São Paulo.

Tratamentos: 6 , com 10 repetições, totalizando 60 ninhos vivos. As alturas médias por tratamento eram: A) $64,5 \mathrm{~cm}$; B) $71,5 \mathrm{~cm}$; C) $63,5 \mathrm{~cm}$;

TABELA 1 - Combate ao cupim de monte: tratamentos, formulações, concentrações e consumo de material. São Pedro, SP, 26 de fevereiro de 1993.

\begin{tabular}{llllc}
\hline Tratamento & \multicolumn{2}{c}{$\begin{array}{l}\text { Produto Comercial (1), } \\
\text { Formulação e } \\
\text { Concentração de IA }\end{array}$} & & \multicolumn{2}{c}{$\begin{array}{c}\text { Produto Comercial } \\
\text { em 100 l } \\
\text { de água }\end{array}$} & por ninho \\
\hline A) Abamectina & & & \\
B) Silafluofem & Vertimec 18CE & CE 1,8\% & $50 \mathrm{~cm}^{3}$ & $0,5 \mathrm{~cm}^{3}$ \\
C) Silafluofem & Hoe 084498 & CE $80 \%$ & $200 \mathrm{~cm}^{3}$ & $2 \mathrm{~cm}^{3}$ \\
D) Fipronil & Hoe 084498 & CE $80 \%$ & $400 \mathrm{~cm}^{3}$ & $4 \mathrm{~cm}^{3}$ \\
E) Fipronil & Regent 20G & G $2 \%$ & - & $15 \mathrm{~g}$ \\
F) Clorpirifós & Regent 20G & G $2 \%$ & - & $20 \mathrm{~g}$ \\
\hline
\end{tabular}

(1) - CE: concentrado emulsionável; G: granulado.

(2) - Ingrediente ativo.

(3) - Tratamento padrão.

IA por ninho: A- 0,009g; B- $1,6 \mathrm{~g}$; C- 3,2g; D- $0,3 \mathrm{~g}$; E- 0,4g; F-0,0375g. 
TABELA 2 - Combate ao cupim de monte: tratamentos, formulações comerciais, concentrações de ingrediente ativo e consumo de material. Piracicaba, SP, 8 e 9 de setembro de 1993.

\begin{tabular}{|c|c|c|c|c|}
\hline \multirow[t]{2}{*}{ Tratamento } & \multirow{2}{*}{\multicolumn{2}{|c|}{$\begin{array}{l}\text { Produto Comercial }{ }^{(1)} \text {, } \\
\text { Formulação e Concentração } \\
\qquad \operatorname{de~} \mathbf{I A}^{(2)}\end{array}$}} & \multicolumn{2}{|c|}{ Produto Comercial } \\
\hline & & & $\begin{array}{l}\text { em } 1001 \\
\text { de água }\end{array}$ & por ninho \\
\hline A- Fipronil & Regent $20 \mathrm{G}$ & G $2 \%$ & $\ldots$ & $10 \mathrm{~g}$ \\
\hline B- Fipronil ${ }^{(3)}$ & Regent $20 \mathrm{G}$ & G $2 \%$ & - & $15 \mathrm{~g}$ \\
\hline C- Bendiocarbe & sem nome & G $0,1 \%$ & $\ldots$ & $20 \mathrm{~g}$ \\
\hline D- Bendiocarbe & sem nome & G $0,5 \%$ & $\ldots$ & $20 \mathrm{~g}$ \\
\hline E- Imidaclopride & Confidor 700 & G $70 \%^{(4)}$ & $15 \mathrm{~g}$ & $0,15 \mathrm{~g}$ \\
\hline F- Imidaclopride & Confidor 700 & G $70 \%$ & $30 \mathrm{~g}$ & $0,30 \mathrm{~g}$ \\
\hline
\end{tabular}

(1) - G: granulado.

(2) - Ingrediente ativo.

(3) - Tratamento padrão.

(4) - Confidor $700 \mathrm{GrDA}$ é granulado dispersível na água.

IA por ninho: A- 0,2g; B- 0,3g; C- $0,02 \mathrm{~g} ;$ D- $0,10 \mathrm{~g} ; \mathrm{E}-0,105 \mathrm{~g} ; \mathrm{F}-0,210 \mathrm{~g}$.

D) $66,0 \mathrm{~cm}$; E) $67,0 \mathrm{~cm}$; F) $81,0 \mathrm{~cm}$; sendo a média geral $68,9 \mathrm{~cm}$. Produtos, formulações, concentrações e consumo de material estão na TABELA 2.

Abertura do cunal: utilizou-se varão de aço e marreta, conforme descrito anteriormente.

Aplicação: realizada em 08 e 09 de setembro de 1993. Em E,F, os granulados foram misturados com água. Todos os ingredientes foram lançados diretamente no núcleo.

TABELA 3 - Combate ao cupim de monte: resultados obtidos. Piracicaba, SP, 10 de junho de 1993.

\begin{tabular}{lcccc}
\hline Tratamento & Ninhos & \multicolumn{2}{c}{ Resultados } \\
\cline { 2 - 5 } & & Vivos & Mortos & $\begin{array}{c}\text { Mortali- } \\
\text { dade(\%) }\end{array}$ \\
\hline A) Abamectina (1) & 10 & 1 & 9 & 90 \\
B) Silafluofem & 10 & 7 & 3 & 30 \\
C) Silafluofem & 10 & 6 & 4 & 40 \\
D) Fipronil & 10 & 1 & 9 & 90 \\
E) Fipronil & 10 & 0 & 10 & 100 \\
F) Clorpirifós & 10 & 10 & 0 & 0 \\
\hline
\end{tabular}

(1) Tratamento padrão.

Sci. agric., Piracicaba, 51(3):505-508, set./dez., 1994
Abertura dos montes: realizada em 04 de fevereiro de 1994, ou seja 148 e 149 dias após a aplicação dos produtos. A presença de $C$. cumulans, ainda que em pequena quantidade, fazia com que a colônia fosse considerada viva; não havendo, era considerada morta.

TABELA 4 - Combate ao cupim de monte : resultados obtidos. Piracicaba, SP, 04 de fevereiro de 1994.

\begin{tabular}{|c|c|c|c|c|}
\hline \multirow[t]{2}{*}{ Tratamento } & \multirow[t]{2}{*}{ Ninhos } & \multicolumn{3}{|c|}{ Resultados } \\
\hline & & Vivos & Mortos & $\begin{array}{l}\text { Mortali- } \\
\text { dade(\%) }\end{array}$ \\
\hline A) Fipronil & 10 & $\mathbf{0}$ & 10 & 100 \\
\hline B) Fipronil ${ }^{(1)}$ & 10 & $\mathbf{0}$ & 10 & 100 \\
\hline C) Bendiocarbe & 10 & 10 & 0 & 0 \\
\hline D) Bendiocarbe & 10 & 7 & 3 & 30 \\
\hline E) Imidaclopride & 10 & 2 & 8 & 80 \\
\hline F) Imidaclopride & le 10 & 0 & 10 & 100 \\
\hline
\end{tabular}

(1) Tratamento padrão.

\section{RESULTADOS E DISCUSSÃO}

Experimento I: Os melhores foram o fipronil e abamectina. $O$ fipronil conseguiu não só atingir, como também superar o nível de eficiência do 
padrão (abamectina). $O$ silafluofem teve eficiência bastante reduzida ( $30 \%$ e $40 \%$ ) e o clorpirifos mostrou-se ineficiente. Ver porcentagens de eficiência na TABELA 3.

Experimento II: O fipronil e a imidaclopride $(0,3 \mathrm{~g})$ foram muito eficazes. A imidaclopride $(0,15 \mathrm{~g})$ resultou numa eficiência menor. $O$ bendiocarbe não apresentou resultados satisfatórios $(0 \%$ de mortalidade na concentração de $0,10 \%$ e $30 \%$ de mortalidade na concentração de $0,5 \%$ ). TABELA 4.

\section{CONCLUSÕES}

Sob as condições em que os trabalhos foram realizados e conforme os resultados obtidos, conclui-se:

Experimento I - foram muito eficientes a abamectina $\mathrm{e}$ as duas dosagens de fipronil. Resultados inexpressivos foram obtidos com o silafluofem e clorpirifós.

Experimento II - foram muito boas as duas dosagens de fipronil e a mais alta de imidaclopride: entretanto, a mais baixa de imidaclopride salientouse um pouco menos e os resultados conseguidos pelo bendiocarbe foram péssimos.

\section{OBSERVAÇÕES FINAIS}

Não houve cupinzeiros marcados sem nenhum tratamento (testemunha): quando os ninhos são bem escolhidos vivem, ao que se acredita, mais de dois anos da data de marcação do campo. Testemunhas tratadas com água também não foram estabelecidas: em INTRODUÇÃO ver HAMAMURA et al. (1990) e MARICONI et al. (1990) a respeito da ineficiência da água.

\section{REFERÊNCIAS BIBLIOGRÁFICAS}

AMANTE, E. Ensaio de campo para controlar o cupim de monte Cornitermes cumulans (Kollar, 1832) (Isoptera, Termitidae). Divulgação Agronômica, Rio de Janeiro, v.9, p.30-33, 1963.

BIONDO, C.J.; GERALDI, F.I.; CLARI, A.I.; DONATONI, J.L.; ARASHIRO, F.Y.; RAIZER, A.J.; MARICONI, F.A.M. Cupim de monte Cormitermes cumulans (Kollar, 1832): combate experimental com formulações inseticidas líquidas. Anais da Escola Superior de Agricultura "Luiz de Queiroz", Piracicaba, v.45, n.1, p.91-97, 1988.
HAMAMURA, R.; RANGEL, R.C.; REGITANO, E.B.; MESQUITA, L.F.; CARDOSO, M.A.C.; CLARI, A.I.; MARICONI, F.A.M. Ensaio de combate ao cupim de monte Comitermes cumulans (Kollar, 1832) com clorpirifós, fentiom e água. Revista de Agricultura, Piracicaba, v.65, n.2, p.195-201, 1990.

MARICONI, F.A.M.; CORREIA, L.S.; ANDRADE, M.E.R.; CARVALHO FILHO, H.C. Combate ao cupim de monte Comitermes cumulans (Kollar, 1832) com inseticidas e diferentes métodos. $\mathbf{O}$ Solo, Piracicaba, v.63, n.1, p.21-31, 1971.

MARICONI, F.A.M.; GERALDI, F.I.; BIONDO, C.J.; DONATONI, J.L.; CLARI, A.I.; ARASHIRO, F.Y.; RAIZER, A.J. Fentiom e clorpirifós no combate ao cupim de monte Comitermes cumulans (Kollar, 1832) (Isoptera Termitidae). Anais da Escola Superior de Agricultura "Luiz de Queiroz", Piracicaba, v.46, n.2, p.295-302, 1989.

MARICONI, F.A.M.; RAIZER, A.J.; ARASHIRO, F.Y.; BIONDO, C.J.; CLARI, A.I. Combate experimental ao cupim de monte Comitermes cumulans (Kollar, 1832) (Isoptera, Termitidae). Anais da Sociedade Entomologica do Brasil, Porto Alegre, v.19, n.1, p.163-169, 1990.

MOTTA, R.; RAIZER, A.J.; SILVA, J.M.; SUGAHARA, C.A.; ARASHIRO, F.Y.; MARICONI, F.A.M. Ensaio de combate a ninhos do cupim Comitermes cumulans (Kollar, 1832). Anais da Escola Superior de Agricultura "Luiz de Queiroz", Piracicaba, v.44, p.1389-1395, 1987.

NAKANO, O.; PROSPERO, A.O. Efeito comparativo de diversos inseticidas no controle do cupim de montículo Cornitermes cumulans (Kollar, 1832). Revista de Agricultura, Piracicaba, v.44, n.2-3, p.98-102, 1969.

SUGAHARA, C.A.; RAIZER, A.J.; MOTTA, R.; ARASHIRO, F.Y.; SILVA, J.M.; MARICONI, F.A.M. Combate experimental ao cupim Comitermes cumulans (Kollar, 1832) em pastagem. Anais da Escola Superior de Agricultura "Luiz de Queiroz", Piracicaba, v.44, p.1381-1387, 1987.

VANETTI, F. Combate ao cupim de montículo Comitermes cumulans (Kollar). Revista Ceres, Viçosa, v.10, n.60, p.437-443, 1959.

Recebido para publicação em 28.04.94 Aceito para publicação em 10.10 .94 